\title{
Circular Arc Representation based on Parametric Complex Functions
}

\author{
Xuexiang Li \\ School of Software, Zhengzhou University, \\ Zhengzhou, China \\ Email: 1xx@zzu.edu.cn
}

\author{
Junxiao Xue \\ School of Software, Zhengzhou University, \\ Zhengzhou, China
}

\begin{abstract}
In CNC machinery, the industrial products and machinery parts are often represented by circular arcs. A new method for representing circular arc based on parametric complex functions is presented in the paper. Since cross ratio is an invariant of the group of all Möbius transformations, and a Möbius transformation maps a circle into another circle, we represent a segment of circular arc with a parametric complex rational function. The representation has no weight factors or control parameters, and it is geometric and affine invariant. Compared with the classical method for representing circular arc, such as NURBS or C-curves, the presented method is much simpler.
\end{abstract}

\section{Keywords -CNC; Circular arc; Möbius transformation}

\section{INTRODUCTION}

Shape represented by two arcs is often required in industrial products and machinery parts $[1,2,5]$. These shapes are designed unambiguous by drawing, and need higher accuracy in manufacturing. Many industrial products and machinery parts are often represented by circular arcs. Currently, most of mechanical parts are processed by linear and circular interpolation. In adition, circular splines has important applications in the design of the robot walking path and the reconstruction of threedimensional vascular. Therefore, the research of circular splines has worldwide concerns $[6,7,8,9]$. The free-form curves representation methods such as B-splines have widely applications in computer aided geometric design and industrial product modeling. However, these methods are in trouble for the representation and design of circular arc. Because quadratic curves cannot be represented exactly by B-spline curves, except for parabola. The approximate representation will bring troubles in application, such as make a simple matter been complicated, and bring added errors.

The presence of NURBS [4] gives a good solution to the problem of circular arc representation. It keeps the advantage of B-splines in the description of freeform shapes, and extends the ability of representation for quadratic curves, includes the exact representation of

Provincial Natural Science Foundation (132300410190) :Research on Digital Geometry Processing Technology and its Application Problem based on Conformal Geometry. quadratic curves. Thus, NURBS occupy a dominant position in geometric design of industrial products. ISO (International organization for standardization) issued the

standard for the exchange of product model data in 1991, and make NURBS been the only one mathematics method for the shape repreaentation of industrial products.

Therefore, NURBS also faces many problems like any method. Especially, how to determine the right weights has been troubled by designers and been an unsolved problem.

C-curves [10] based on trigonometric polynomial can deal precisely with circular arcs, but the shape of Ccurves depend on a parameter $\alpha$. However, the selection of parameters is a difficult problem. Meanwhile, rigonometric polynomial may greatly increase the amount of calculation.

A Möbius transformation can be obtained by first performing stereographic projection from the plane to the unit two-sphere, rotating and moving the sphere to a new location and orientation in space, and then performing stereographic projection (from the new position of the sphere) to the plane. These transformations preserve angles, map every straight line to a line or circle, and map every circle to a line or circle.

The Möbius transformations are projective transformations of the complex projective line. They form a group called the Möbius group which is the projective linear group $\operatorname{PGL}(2, \mathrm{C})$. Together with its subgroups, it has numerous applications in mathematics and physics.

Möbius transformations are named in honor of August Ferdinand Möbius; they are also variously named homographies, homographic transformations, linear fractional transformations, bilinear transformations, or fractional linear transformations.

In physics, the identity component of the Lorentz group acts on the celestial sphere in the same way that the Möbius group acts on the Riemann sphere. In fact, these two groups are isomorphic. An observer who accelerates to relativistic velocities will see the pattern of constellations as seen near the Earth continuously transform according to infinitesimal Möbius transformations. This observation is often taken as the starting point of twistor theory. 
Certain subgroups of the Möbius group form the automorphism groups of the other simply-connected Riemann surfaces (the complex plane and the hyperbolic plane). As such, Möbius transformations play an important role in the theory of Riemann surfaces. The fundamental group of every Riemann surface is a discrete subgroup of the Möbius group (see Fuchsian group and Kleinian group). A particularly important discrete subgroup of the Möbius group is the modular group; it is central to the theory of many fractals, modular forms, elliptic curves and Pellian equations.

Möbius transformations can be more generally defined in spaces of dimension $n>2$ as the bijective conformal orientation-preserving maps from the n-sphere to the nsphere. Such a transformation is the most general form of conformal mapping of a domain. According to Liouville's theorem a Möbius transformation can be expressed as a composition of translations, similarities, orthogonal transformations and inversions.

Möbius transformations [3] (linear fractional transformations) are one-to-one, onto and conformal (angle preserving) maps of the so-called extended complex plane. We will develop the basic properties of these maps and classify the one-to-one and onto conformal maps of the unit disk and the upper half plane using the symmetry principle. A new method for representing circular arc based on parametric complex functions is presented in the paper. Since cross ratio is an invariant of the group of all Möbius transformations, and a Möbius transformation maps a circle into another circle, we represent a segment of circular arc with a parametric complex rational function. The representation has no weight factors or control parameters, and it is geometric and affine invariant. Compared with the classical method for representing circular arc, such as NURBS or Ccurves, the presented method is much simpler.

\section{MÖBIUS TRANSFORMATION}

\section{A. Definition}

Definition 2.1 The general form of a Möbius transformation is given by

$$
f(z)=\frac{a z+b}{c z+d}
$$

Where $a, b, c, d$ are any complex numbers satisfying $a d-b c \neq 0$. (If $a d=b c$ the rational function defined above is a constant and is not considered a Möbius transformation.)

In case $c \neq 0$ this definition is extended to the whole Riemann sphere by defining

$$
f(-d / c)=\infty \text { and } f(\infty)=a / c
$$

if $c=0$ we define $f(\infty)=\infty$. This turns $f(z)$ into a bijective holomorphic function from the Riemann sphere to the Riemann sphere.

\section{B. Properties of Möbius transformations}

Möbius transformation is conformal at every point except its pole.

Möbius transformation maps the class of circled and lines to itself.

From this decomposition, we see that Möbius transformations carry over all non-trivial properties of circle inversion. For example, the preservation of angles is reduced to proving that circle inversion preserves angles since the other types of transformations are dilation and isometries (translation, reflection, rotation), which trivially preserve angles.

Furthermore, Möbius transformations map generalized circles to generalized circles since circle inversion has this property. A generalized circle is either a circle or a line, the latter being considered as a circle through the point at infinity. Note that a Möbius transformation does not necessarily map circles to circles and lines to lines: it can mix the two. Even if it maps a circle to another circle, it does not necessarily map the first circle's center to the second circle's center.

Cross-ratios are invariant under Möbius transformations. That is, if a Möbius transformation maps four distinct points $z_{1}, z_{2}, z_{3}, z_{4}$ to four distinct points $w_{1}, w_{2}, w_{3}, w_{4}$ respectively, then

$$
\frac{\left(z_{1}-z_{3}\right)\left(z_{2}-z_{4}\right)}{\left(z_{2}-z_{3}\right)\left(z_{1}-z_{4}\right)}=\frac{\left(w_{1}-w_{3}\right)\left(w_{2}-w_{4}\right)}{\left(w_{2}-w_{3}\right)\left(w_{1}-w_{4}\right)}
$$

If one of the points $z_{1}, z_{2}, z_{3}, z_{4}$ is the point at infinity, then the cross-ratio has to be defined by taking the appropriate limit; e.g. the cross-ratio of $z_{1}, z_{2}, z_{3}, \infty$ is

$$
\frac{\left(z_{1}-z_{3}\right)}{\left(z_{2}-z_{3}\right)}
$$

The cross ratio of four different points is real if and only if there is a line or a circle passing through them. This is another way to show that Möbius transformations preserve generalized circles.

Four distinct points $z_{1}, z_{2}, z_{3}, z_{4}$ on complex plane in the same circle If and only if their cross ratio for real.

Suppose three points $z_{1}, z_{2}, z_{3}$ in Z-plane are transformed into three points $w_{1}, w_{2}, w_{3}$ in $\mathrm{W}$-plane by Möbius transformation. That is $w_{j}=f\left(z_{j}\right),(j=1,2,3)$. The Möbius transformation is uniquely determined, and can be written in the following form

$$
w=f(z)=\frac{\left(z-z_{3}\right)\left(z_{1}-z_{2}\right) w_{2}\left(w_{1}-w_{3}\right)-\left(z-z_{2}\right)\left(z_{1}-z_{3}\right) w_{3}\left(w_{1}-w_{2}\right)}{\left(z-z_{3}\right)\left(z_{1}-z_{2}\right)\left(w_{1}-w_{3}\right)-\left(z-z_{2}\right)\left(z_{1}-z_{3}\right)\left(w_{1}-w_{2}\right)}
$$




\section{CIRCUlAR ARC REPRESENTATION}

We know that any three points $V_{1}\left(x_{1}, y_{1}\right), V_{2}\left(x_{2}, y_{2}\right), V_{3}\left(x_{3}, y_{3}\right)$ which are not in the same line can determine a circle. Therefore, we could regard the three points $V_{1}\left(x_{1}, y_{1}\right), V_{2}\left(x_{2}, y_{2}\right), V_{3}\left(x_{3}, y_{3}\right)$ as three complex points $w_{j}=w\left(t_{j}\right),(j=1,2,3)$ in complex plane. Then we can use the properties of Möbius transformation to construct an accurate representation of the arc curve determined by the three points by a complex rational functions

$$
\text { Suppose } V_{1}\left(x_{1}, y_{1}\right), V_{2}\left(x_{2}, y_{2}\right), V_{3}\left(x_{3}, y_{3}\right) \text { are }
$$

three points which are not in the same line, and

$$
w_{j}=x_{j}+i y_{j}(j=1,2,3)
$$

Then (7) accurately represent a section of circular arc curve is defined by three points. and satisfy $w_{j}=w\left(t_{j}\right),(j=1,2,3)$. Where, $t_{j} \in R(j=1,2,3)$ are three real nodes.

$w(t)=\frac{\left(t-t_{3}\right)\left(t_{1}-t_{2}\right) w_{2}\left(w_{1}-w_{3}\right)-\left(t-t_{2}\right)\left(t_{1}-t_{3}\right) w_{3}\left(w_{1}-w_{2}\right)}{\left(t-t_{3}\right)\left(t_{1}-t_{2}\right)\left(w_{1}-w_{3}\right)-\left(t-t_{2}\right)\left(t_{1}-t_{3}\right)\left(w_{1}-w_{2}\right)}, t \in\left[t_{1}, t_{3}\right]$

1)

$$
t_{1}<t_{2}<t_{3} .
$$

Proof: we could regard the parameter $t$ in formula (1) as a complex number, and its imaginary part is zero. Obviously, formula (1) determines a Möbius transformation. By theorem 2.3, the cross-ratios of $w, w_{1}, w_{2}, w_{3}$ is equal to the cross-ratios of $t, t_{1}, t_{2}, t_{3}$. The cross-ratios of $t, t_{1}, t_{2}, t_{3}$ is real, so the cross-ratios of $w, w_{1}, w_{2}, w_{3}$ is also real. By theorem 2.4 , the points $w, w_{1}, w_{2}, w_{3}$ are on the same circumference. Thus, the function of formula (1) represent a arc curve which is detemined by the three points $w_{1}, w_{2}, w_{3}$.

In fact, the following description formula (1) is non degenerate. formula (1) can be sorted as the standard form

$$
w(t)=\frac{a t+b}{c t+d}
$$

Where

$$
\begin{aligned}
& a=\left(t_{1}-t_{2}\right) w_{2}\left(w_{1}-w_{3}\right)-\left(t_{1}-t_{3}\right) w_{3}\left(w_{1}-w_{2}\right), \\
& b=t_{2}\left(t_{1}-t_{3}\right) w_{3}\left(w_{1}-w_{2}\right)-t_{3}\left(t_{1}-t_{2}\right) w_{2}\left(w_{1}-w_{3}\right), \\
& c=\left(t_{1}-t_{2}\right)\left(w_{1}-w_{3}\right)-\left(t_{1}-t_{3}\right)\left(w_{1}-w_{2}\right), \\
& d=t_{2}\left(t_{1}-t_{3}\right)\left(w_{1}-w_{2}\right)-t_{3}\left(t_{1}-t_{2}\right)\left(w_{1}-w_{3}\right), \\
& t_{1}<t_{2}<t_{3}, t \in\left[t_{1}, t_{3}\right]
\end{aligned}
$$

Because

$a d-b c=\left(t_{1}-t_{2}\right)\left(w_{1}-w_{2}\right)\left(t_{2}-t_{3}\right)\left(w_{2}-w_{3}\right)\left(t_{1}-t_{3}\right)\left(w_{1}-w_{3}\right) \neq 0$

So formula (1) is non degenerate.

We denote formula (1) as complex rational curves, the following will discuss some properties of it.

\section{PROPERTIES OF PARAMETRIC COMPLEX RATIONAL CURVES}

We give a representation of circular arc curve Möbius transformation in the previous section. In our method, the arc curve is represented by a complex rational function with parameters. In following, we will discuss the properties of the omplex rational function with parameters

\section{A. Affine invariance}

Suppose

$$
\begin{aligned}
\Phi: R^{2} & \rightarrow R^{2} \\
w & \mapsto w \cdot M+N
\end{aligned}
$$

Where: $M$ is a $2 \times 2$ matrix and $N$ is a $1 \times 2$ vector. Substitute $\Phi\left(w_{j}\right)=w_{j} \cdot M+N$ in formula (1), We can get the following result after a simple calculation: $w\left(t ; w_{1} \cdot M+N, w_{2} \cdot M+N, w_{3} \cdot M+N\right)=w\left(t ; w_{1}, w_{2}, w_{3}\right) \cdot M+N$ .That is

$$
w\left[t ; \Phi\left(w_{1}\right), \Phi\left(w_{2}\right), \Phi\left(w_{3}\right)\right]=\Phi\left[w\left(t ; w_{1}, w_{2}, w_{3}\right)\right]
$$

Formula (5) shows that the location and the shape of the parametric complex rational arc curve is only rely on the points $V_{1}, V_{2}, V_{3}$. It is not depend on the choice of the coordinate system, which is the geometric invariance. In addition, formula (5) also shows that change of the parameter and the nods in formula (1) does not affect the position and the shape of the curve. Therefore, we can according to actual needs, select the appropriate. The parameters, such as arc length parameterization. The parameters are taken as the arc length parameter, and the nodesvalues from the corresponding Circular arc length to determine The subject selection, namely has the geometric invariance.

\section{B. Geometric invariance Suppose}

$$
\begin{aligned}
\varphi: & R \rightarrow R, \\
& t \mapsto k t+h
\end{aligned}
$$

Where $k, h$ are real. 
Substitute

$\varphi(t)=k t+h, \varphi\left(t_{j}\right)=k t_{j}+h,(j=1,2,3)$

formula (1), we can get the following result after a simple calculation:

$$
w\left[\varphi(t) ; \varphi\left(t_{1}\right), \varphi\left(t_{2}\right), \varphi\left(t_{3}\right)\right]=w(t)
$$

These characteristics indicate that the parameters defining the interval and node is not essential, you can select the appropriate parameters to define the interval and node according to the needs. For convenience, we may make

$$
t_{1}=0, t_{2}=\frac{1}{2}, t_{3}=1 .
$$

Parameters are defined in the interval $[0,1]$.

So the arc curvecan be expressed as the following form:

$$
w(t)=\frac{(t-1) w_{2}\left(w_{1}-w_{3}\right)-(2 t-1) w_{3}\left(w_{1}-w_{2}\right)}{(t-1)\left(w_{1}-w_{3}\right)-(2 t-1)\left(w_{1}-w_{2}\right)}, t \in[0,1]
$$

\section{Differential properties}

According to formula (2) ana (3)

$$
\begin{gathered}
\frac{d w}{d t}=\frac{a d-b c}{(c t+d)^{2}} \\
=\frac{\left(t_{1}-t_{2}\right)\left(w_{1}-w_{2}\right)\left(t_{2}-t_{3}\right)\left(w_{2}-w_{3}\right)\left(t_{1}-t_{3}\right)\left(w_{1}-w_{3}\right)}{\left[\left(t-t_{3}\right)\left(t_{1}-t_{2}\right)\left(w_{1}-w_{3}\right)-\left(t-t_{2}\right)\left(t_{1}-t_{3}\right)\left(w_{1}-w_{2}\right)\right]^{2}}
\end{gathered}
$$

so,

$$
\begin{aligned}
& \left.\frac{d w}{d t}\right|_{t=t_{1}}=\frac{t_{2}-t_{3}}{\left(t_{1}-t_{3}\right)\left(t_{1}-t_{2}\right)} \cdot \frac{\left(w_{1}-w_{2}\right)\left(w_{1}-w_{3}\right)}{w_{2}-w_{3}} \\
& \left.\frac{d w}{d t}\right|_{t=t_{3}}=\frac{t_{1}-t_{2}}{\left(t_{1}-t_{3}\right)\left(t_{2}-t_{3}\right)} \cdot \frac{\left(w_{1}-w_{3}\right)\left(w_{2}-w_{3}\right)}{w_{1}-w_{2}}
\end{aligned}
$$

If

$$
t_{1}=0, t_{2}=\frac{1}{2}, t_{3}=1 \text {, }
$$

then:

$$
\begin{aligned}
& w^{\prime}(0)=-\frac{\left(w_{1}-w_{2}\right)\left(w_{1}-w_{3}\right)}{w_{2}-w_{3}} \\
& w^{\prime}(1)=-\frac{\left(w_{1}-w_{3}\right)\left(w_{2}-w_{3}\right)}{w_{1}-w_{2}}
\end{aligned}
$$

\section{CONCLUSION}

A new method for representing circular arc based on parametric complex functions is presented in the paper. Since cross ratio is an invariant of the group of all Möbius transformations, and a Möbius transformation maps a circle into another circle, we represent a segment of circular arc with a parametric complex rational function. The representation has no weight factors or control parameters, and it is geometric and affine invariant. Compared with the classical method for representing circular arc, such as NURBS or C-curves, the presented method is much simpler.

\section{REFERENCES}

[1] Wang Qi, Guo Fei, Wang Qiyi, Circular arc spline approximation for auto programming profiles of machine parts [J], Chinese Journal of Mechanical Engineering, 1998, 34(2): 20-25.

[2] Lv Yonggang, Wang Guozhao, and et al, Single arc spline shape preserving interpolation algorithm [J], Chinese Journal of computers, 2002, 25(5): 483-486.

[3] L. V. Ahlfors, Complex Alalysis[M], New York: McGraw-Hill Book Co., 1979.

[4] Shi Fazhong, Computer aided geometric design and non uniform rational $\mathrm{B}$ spline $[\mathrm{M}]$, Beijing: Beihang University press, 1994.

[5] Zhu Xiaolin, A parametric rational arc spline [J], Journal of HeFei University of Technology (NATURAL SCIENCE EDITION), 2003, 26(2): 180-184

[6] [M. Yeung and D. J. Walton, Curve fitting with arcsplines for $\mathrm{NC}$ tool path generation[J], Computer-Aided Design, 1994, 26(11): 845-849.

[7] Z. Li and D. S. Meek, Smoothing an Arc Spline[J], Computers \& Graphics, 2005, 29(4): 576-587.

[8] D. S. Meek and D. J. Walton, The family of biarcs that matches planar, two-point G1 Hermite data[J], Journal of Computational and Applied Mathematics, 2008, 212(1): 31-45.

[9] R.L. Scot Drysdale and G “ unter Rote and Astrid Sturm, Approximation of an open polygonal curve with a minimum number of circular arcs and biarcs[J], Computational Geometry: Theory and Applications[J], 2008, 41(1-2): 31-47.

[10] J. W. Zhang, C-curves: an extension of cubic curves[J], Computer Aided Geometric Design,1996, 13(3): 199-217. 